\title{
BALLISTIC PHASE PERFORMANCE MODEL FOR COLD GAS ATTITUDE CONTROL IN CRYOGENIC UPPER STAGES
}

\author{
M. Werner, S. Kottmeier, T. Bruns, and N. Korus \\ Airbus Defence and Space \\ 1 Airbus-Allee, Bremen 28199, Germany
}

During the ballistic phase of a cryogenic upper stage, attitude and orbit control are usually performed by a cold gas thruster system. Since the thruster inlet pressure is driven by the propellant tank ullage conditions, a sufficient pressure regeneration rate has to be assured to compensate the pressure decrease during thruster activation. The coupling between the thruster activation sequence, stage kinematics, and thermal environment is essential for the performance prediction of cold gas systems. An iteration loop using a statistical model considering the thruster activation sequence is applied. For detailed final analysis, an EcosimPro model is used, including three-dimensional (3D) CFD (computational fluid dynamics) sloshing analysis and all relevant boundary conditions.

\section{NOMENCLATURE}

$c_{\mathrm{pl}} \quad$ Specific heat of liquid, $\mathrm{J} /(\mathrm{kg} \cdot \mathrm{K})$

$C_{\mathrm{sf}}$ Surface factor

$C_{\ddot{x}} \quad$ Acceleration coefficient

$d$ Depressurization slope function

$f_{\text {boi }}$ Film boiling factor

$L_{b} \quad$ Capillary length scale of a bubble, $\mathrm{m}$

$M_{n}$ Nozzle fraction coefficient

$n \quad$ Number of activated nozzles

$P \quad$ Pressure, $\mathrm{Pa}$

Pr Prandtl number

$\dot{q} \quad$ Heat flux, $\mathrm{W} / \mathrm{m}^{2}$

$S_{d} \quad$ Depressurization coefficient

$S_{r} \quad$ Regeneration coefficient

$t \quad$ Time, s 
$T_{s} \quad$ Saturation temperature, $\mathrm{K}$

$T_{w} \quad$ Wall temperature, $\mathrm{K}$

$\Delta h_{v}$ Heat of evaporation, $\mathrm{J} / \mathrm{kg}$

$\eta_{l} \quad$ Dynamic viscosity, $\mathrm{N} \cdot \mathrm{s} / \mathrm{m}^{2}$

\section{DEFINITIONS}

FJ Fairing separation

Ho Ignition main stage

H1 Solid rocket booster separation

H2 Main stage shutdown

H3.1 Upper stage shutdown

H4.1 Separation payload 1

H4.2 Separation dual launch adapter

H4.3 Separation payload 2

H5 Stage passivation

K2.1 Upper stage ignition

\section{GLOSSARY}

$\begin{array}{ll}\text { 1D } & \text { One-dimensional } \\ \text { 2D } & \text { Two-dimensional } \\ \text { 3D } & \text { Three-dimensional } \\ \text { ACS } & \text { Attitude Control System } \\ \text { CFD } & \text { Computational Fluid Dynamics } \\ \text { CoG } & \text { Center of Gravity } \\ \text { EL } & \text { EcosimPro Language } \\ \text { EPC } & \text { Cryogenic main core stage (Etage Principal Cryotechnique) } \\ \text { ESC-A } & \text { Cryogenic Upper Stage (Etage Supérieur Cryotechnique Type A) } \\ \text { ESPSS } & \text { European Space Propulsion System Simulation } \\ \text { FiPS } & \text { Final Phase Simulator } \\ \text { FPS } & \text { Functional Propulsion System } \\ \text { FTMCI } & \text { Forces, Torques, Mass, Center of Gravity, Inertias } \\ \text { GH } & \text { Gaseous Hydrogen } \\ \text { GOx } & \text { Gaseous Oxygen } \\ \text { GW } & \text { Gas-Wall Interface } \\ \text { L5xx } & \text { Ariane Launcher Lot No. (Lanceur 5xx) } \\ \text { LG } & \text { Liquid-Gas Interface } \\ \text { LH } & \text { Liquid Hydrogen } \\ \text { LOx } & \text { Liquid Oxygen } \\ \text { LW } & \text { Liquid-Wall Interface }\end{array}$




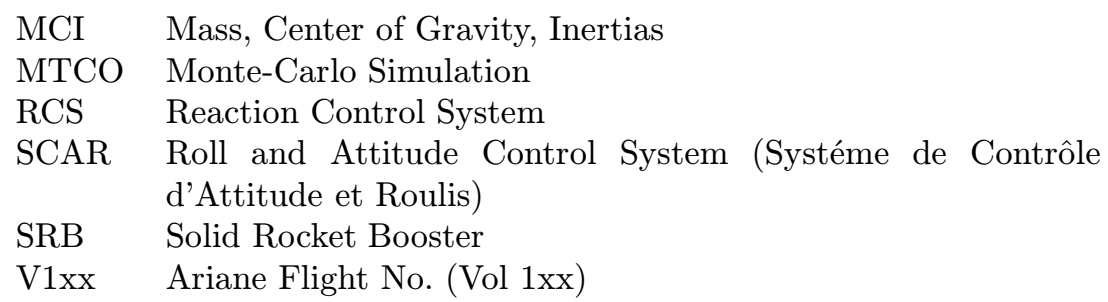

\section{INTRODUCTION}

During liftoff the Ariane 5 launcher is propelled by two solid boosters and the main cryogenic stage. In the course of the ascent phase, the solid boosters and, subsequently, the main stage are separated (Fig. 1). Being the last stage, ESC-A (Etage Supérieur Cryotechnique Type A) completes the mission by performing a last boost and ejecting the satellites in their specific orbit after engine shutdown (H3).
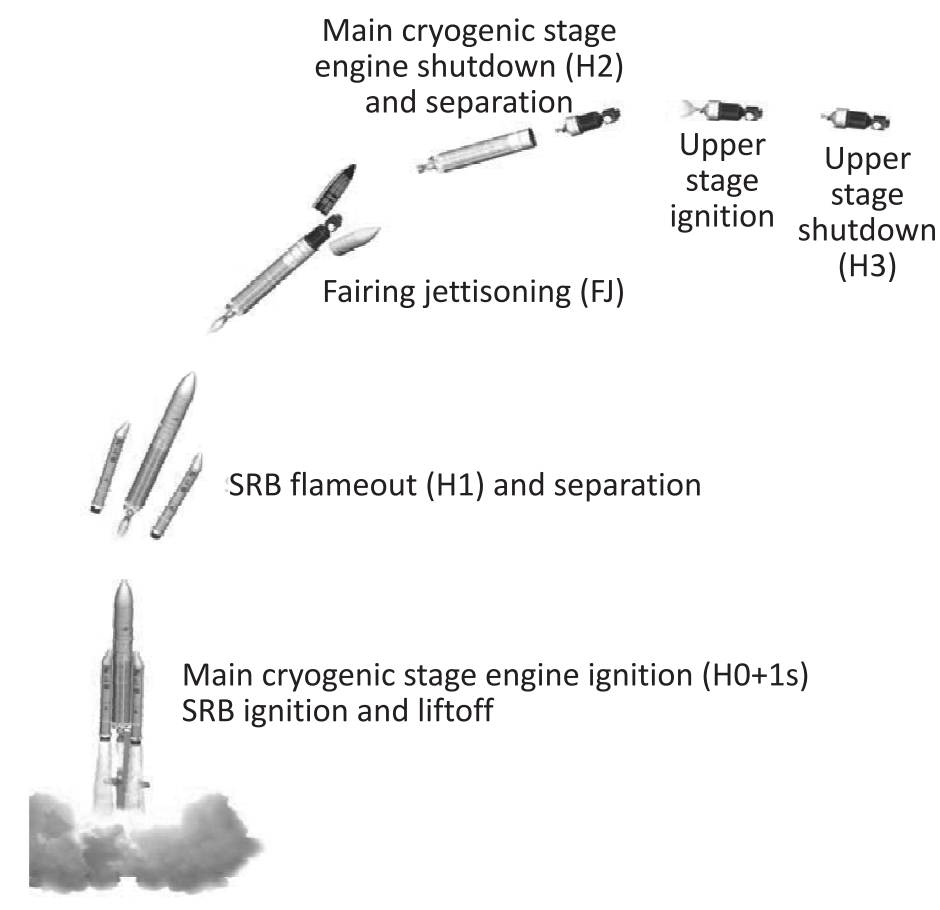

Figure 1 Ariane 5 mission sequence (illustration by Arianespace) 
The ESC-A has a diameter of $5.4 \mathrm{~m}$ and consists of two propellant tanks for liquid hydrogen $\left(\mathrm{LH}_{2}\right)$ and liquid oxygen $(\mathrm{LOx})$ with a volume of $39.4 \mathrm{~m}^{3}$ and $11.4 \mathrm{~m}^{3}$, respectively. A cold gas system is used to provide linear acceleration as well as attitude and roll control during the ballistic phase (also called SCAR (Système de Contrôle d'Attitude et Roulis) phase).

The system splits into hydrogen and oxygen side, which both consist of piping connecting the cryogenic propellant tanks and thruster assemblies. During the ballistic phase, both tanks contain liquid and gaseous residuals. After the engine shutdown, the liquid phases are a volumetrically small part, most of the volume is filled with gas.

Attitude and roll control is provided by the $\mathrm{GH}_{2}$ SCAR system, which consists of four blocks with three nozzles each. Linear acceleration, as needed for distancing and positioning maneuvers, is provided by the oxygen purge and balancing nozzles. The blowout of gaseous oxygen parallel to the stage $x$-axis is controlled by the $\mathrm{S} 34$ valve. Due to different lever arms relative to the stage center of gravity $(\mathrm{CoG})$, the oxygen thrusters are not identical in terms of geometry.

To ensure the sufficient performance of the cold gas thrusters according to the mission profile, the pressure evolution in the propellant tank ullage has to be predicted. This paper describes the iteration loop of thruster activation sequence and provided thrust applying a simplified statistical MATLAB model. Furthermore, the setup of a one-dimensional (1D) EcosimPro system model comprising 3D sloshing effects is described based on the preceding fundamental research $[1,2]$. In the perimeter of this paper, only the $\mathrm{LH}_{2}$ circuit is presented.

\section{ARIANE 5 CRYOGENIC UPPER STAGE SYSTEM OVERVIEW}

To facilitate the understanding of the overall problems connected to the ballistic phase performance prediction, this section gives an overview of the general technical concepts used in the Ariane 5 cryogenic upper stage functional propulsion system (FPS).

The FPS can be divided into the independent $\mathrm{LOx}$ and $\mathrm{LH}_{2}$ circuits with their respective helium pressurization systems, propellant tanks, fluid control equipment, and ACS (attitude control system) components.

The primary component of the hydrogen circuit (Fig. 2) is the hydrogen tank with a diameter of $5.4 \mathrm{~m}$ and a volume of $39.4 \mathrm{~m}^{3}$. The single turbopump feedline inlet is located in the tank lower dome section, causing for a small geometrical $\mathrm{LH}_{2}$ residual after engine shutdown. For the initial tank prepressurization, gaseous helium is used, leading to a volumetrically small helium fraction 


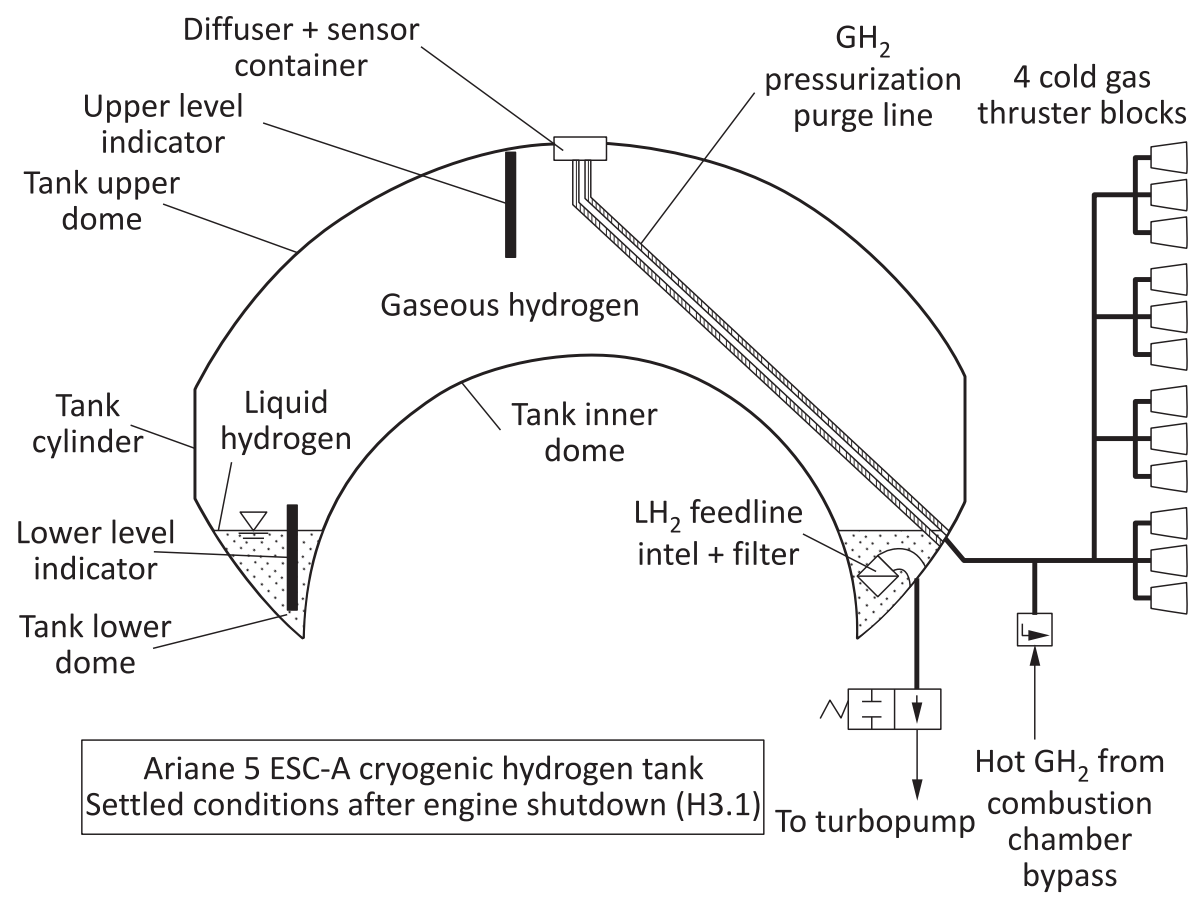

Figure 2 Simplified ESC-A hydrogen circuit

in the ullage gas composition. During the propelled ascent phase, the tank ullage pressure is regulated via a hydrogen bleed flow from the engine cooling system, leading to a relatively hot ullage (approximately $140 \mathrm{~K}$ ) consisting almost entirely of gaseous hydrogen $\left(\mathrm{GH}_{2}\right)$. During the ballistic phase, the turbopump feed and the $\mathrm{GH}_{2}$ repressurization valve are closed and the ullage gas is used in the 12 cold gas thrusters for attitude control. The pressure loss due to thruster activation is regenerated by the natural evaporation of the geometrical $\mathrm{LH}_{2}$ residual.

The primary component of the oxygen circuit (Fig. 3) is the oxygen tank with a diameter of $2.6 \mathrm{~m}$ and a volume of $11.4 \mathrm{~m}^{3}$. The turbopump feedline inlet is located in the bottom center of the tank; so, the LOx residual is driven primarily by diphasic and thermal residuals. During ascent, the tank is pressurized only by gaseous helium from a single high pressure helium tank. After engine shutdown, natural evaporation rates are the driving part in the tank pressure regeneration. The tank ullage consists of a mixture of gaseous helium and oxygen with a defined stratification under accelerated conditions. This mixture ratio plays a major role in the LOx thruster performance prediction. The LOx thrusters are used both 


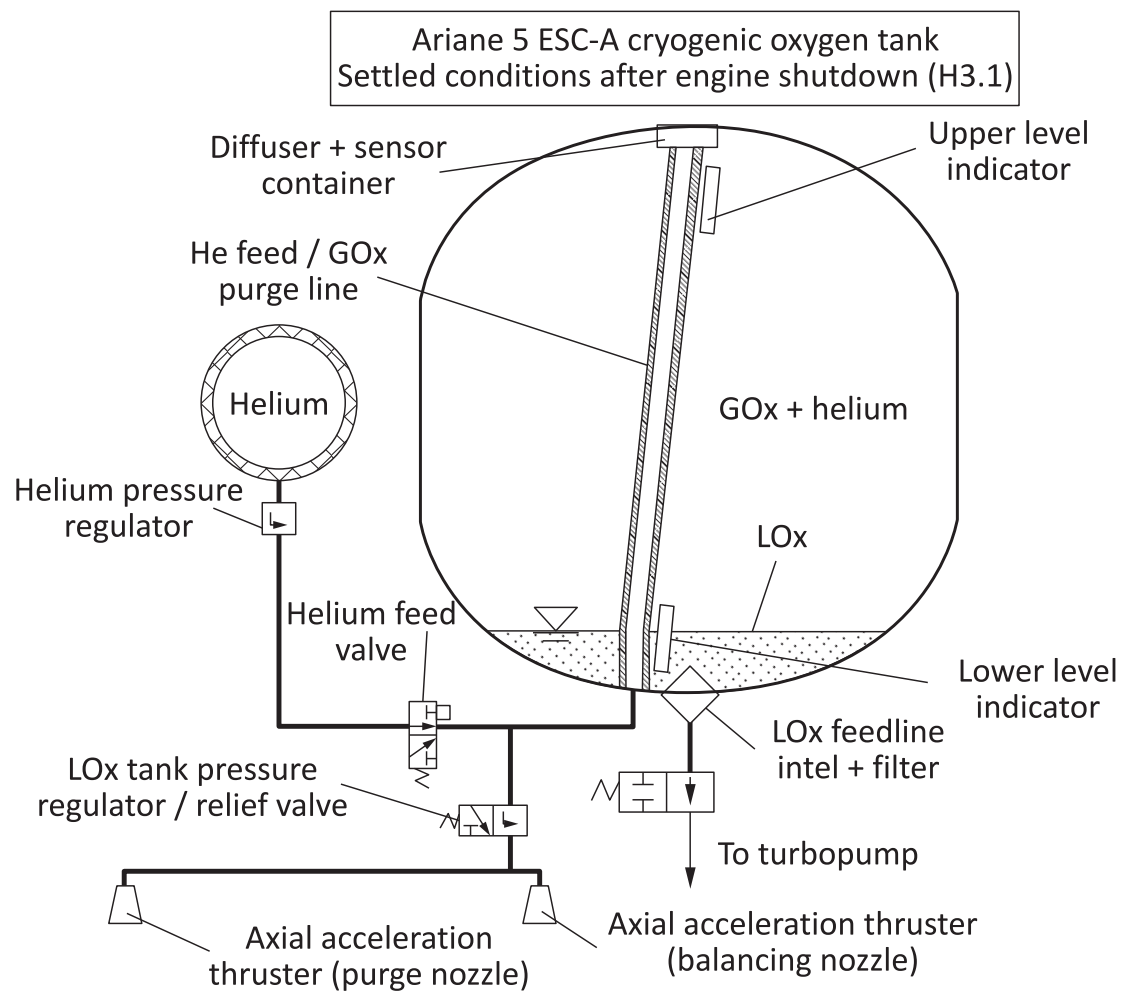

Figure 3 Simplified ESC-A oxygen circuit

for tank pressure regulation and axial acceleration. To compensate induced torques due to the thruster position relative to the stage $\mathrm{CoG}$, the nozzles are not identical in terms of geometry.

\section{BALLISTIC PHASE PERFORMANCE PREDICTION}

In the case of ESC-A acceleration in flight direction (e.g., during boost phase), the propellant is settled at the tank bottom next to the draining outlet. Due to the settled propellant position, the wetted surface areas and, in consequence, the propellant tank conditions are well known.

During the ballistic phase, attitude control maneuvers are performed to place the payloads in their transfer orbits. In this phase, all occurring accelerations are induced by the SCAR, respectively, LOx-purging-system, requiring a certain 


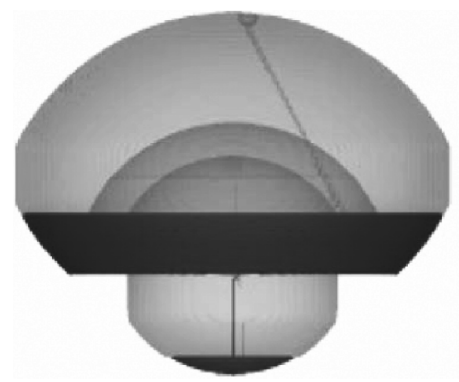

(a)

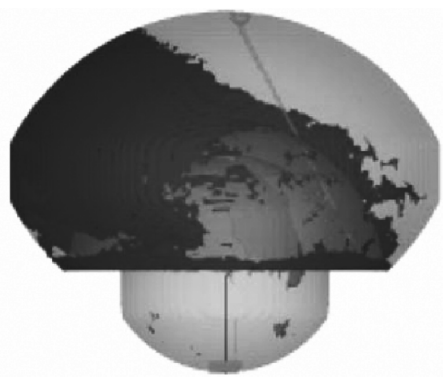

(b)

Figure 4 Examples of settled propellant $(a)$ and sloshing $(b)$

thruster performance. The thrust level is dependent on the provided pressure determined by the propellant tank ullage pressure and the pressure losses as a function of the number of simultaneously activated thrusters. The introduced disturbing accelerations lead to sloshing of the liquid and changing wetted surface areas, affecting the conditions of the propellant tank. Figure 4 illustrates an example of settled and sloshing propellant [3].

At a first glance, the cold gas thruster activation leads to a pressure decrease in the propellant tank. In addition, the alternating states of acceleration and micro-gravity lead to movements of the residual propellant and oxidizer, which cause casual sloshing. With liquid particles moving freely through the ullage volume and increasing the free liquid surface, higher evaporation rates are achieved, leading to disturbances in the ullage pressure.

Figure 5 shows the ullage pressure evolution of the $\mathrm{LH}_{2}$ tank. After engine flameout, a significant pressure drop is visible caused by a stage slewing ma-

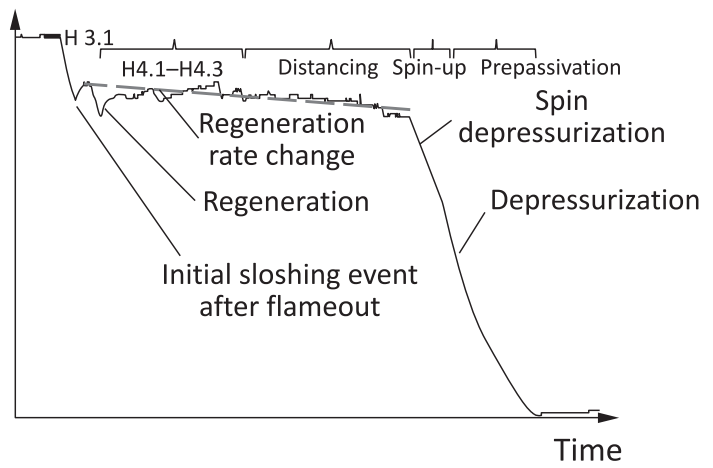

Figure 5 Liquid hydrogen ullage pressure evolution 
neuver, which leads to propellant sloshing. The pressure is regenerating until the next pressure drop is induced. Over the entire ballistic phase, the cooling of the tank structure due to sloshing cannot be compensated by the thermal environment. In consequence, the final pressure regeneration level is decreasing (cf. slope illustrated in Fig. 5).

The pressure evolution in the propellant tank is primarily driven by the evaporation rate of the respective liquid (dependent on the boiling mode) and the gas expulsion through the SCAR. While the mechanisms of the gas expulsion as function of the ullage pressure are relatively well understood, the mechanisms behind the boiling mode are depending on a large range of uncertain parameters connected to the overall system design.

The hydromechanical effects contain all reactions induced by the change of the kinetic state of the stage, bounded by the overall tank, line, and nozzle geometry and the properties of the respective residual fluids. The movement of the fluid and interactions with walls, bulkheads, baffles, and other installations change the absolute free liquid surface, leading to modifications in evaporation rates. Furthermore, the inertia of the moving liquids may disturb the motion of the stage itself leading to additional corrective maneuvers.

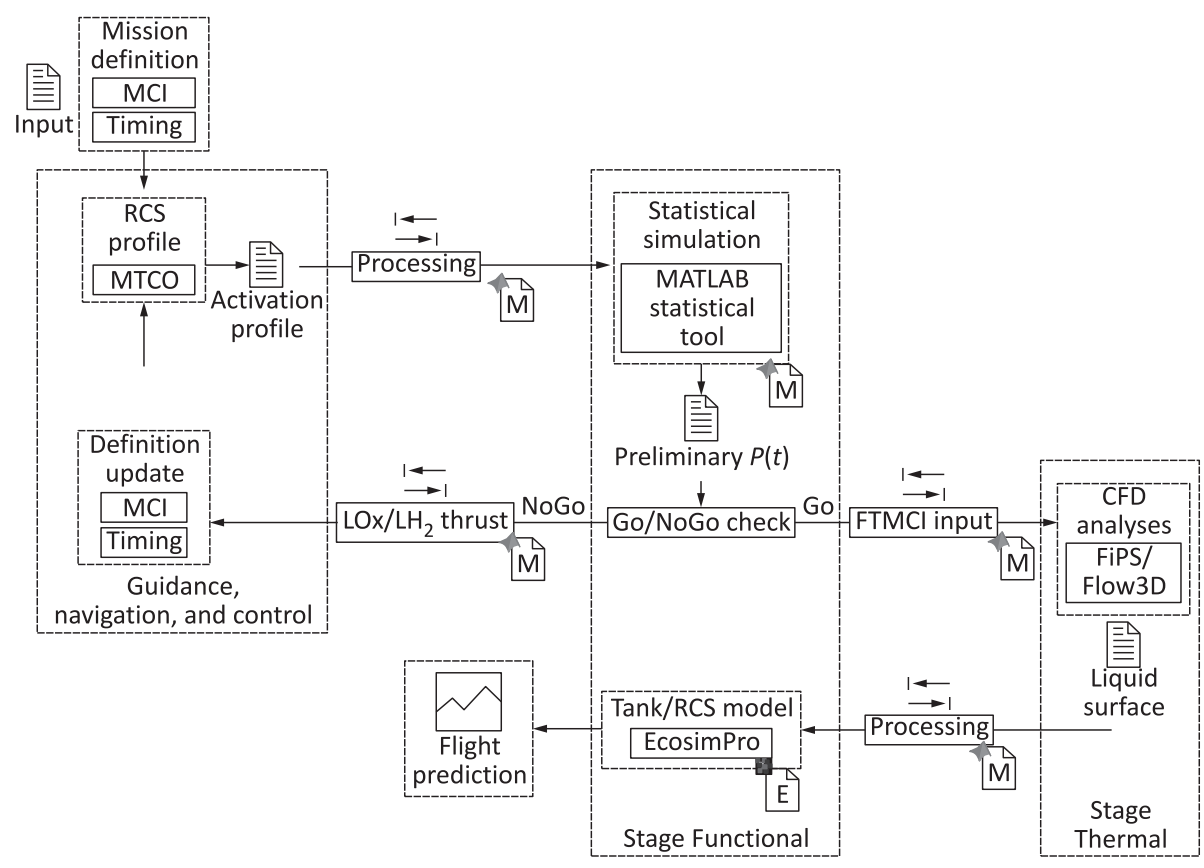

Figure 6 Ballistic flight performance prediction work flow 


\section{ANALYSIS WORK FLOW}

A ballistic phase performance prediction has to cover the complex effects of thermo- and hydrodynamics as well as the interaction with stage kinetics, involving various engineering disciplines and models. To increase the analysis performance by decreasing cycle time and costs, the work flow is separated into a rough iteration loop and an accurate flight prediction model (Fig. 6). The work flow is initiated by the definition of the specific mission phases and the generation of corresponding SCAR activation sequences.

Due to the fact that the activation profiles affect the tank ullage pressure evolution and, in consequence, the thrust level, a consistent mission profile has to be ensured. Therefore, an iterative process of several loops could be necessary for nonstandard missions. A rough numerical model is sufficient because at this point, iteration speed is more important than a high accuracy level. This model is based on a statistical approach and is described later in this paper.

When the iterative loop is successful, i. e., the mission profile with corresponding thruster activation profiles as well as thrust levels and tank ullage pressure evolution are consistent, more accurate numerical models are used for final ballistic phase performance prediction. Due to the consideration of the wide range of physical effects and boundary conditions, the calculation duration is significantly increased in comparison with the rough statistical model.

\section{STATISTICAL PERFORMANCE MODEL}

A wide range of parameters has to be respected in the estimation of the depressurization and regeneration behavior. To limit the analysis effort, it has to be demonstrated that these effects are statistically covered by the number of performed flights and can be simulated in a statistical model by simple correlation mechanisms. For the model, the two main effects, the thermal environment and the stage movement, are taken into account and examined in terms of impact.

\subsection{Numerical Model}

A numerical model for the simulation of the $\mathrm{LH}_{2}$ ullage pressure was set up in MATLAB. The model makes three basic assumptions:

(1) the pressure loss caused by thruster activations can be described as exponential function driven by the parameter activation time $t$ and the number of active nozzles $n$. Further, major influences include the stage acceleration and the point in mission time, resembling the mass of liquid residuals; 
(2) the pressure regeneration in-between thruster activations can be described as exponential function driven by the parameter regeneration time $t$. Further, major influences include the stage acceleration and the point in mission time, resembling the mass of liquid residuals; and

(3) the optimization of the given set of parameters and the validation with a sufficient number of in-flight measurements will cover all unexpected, rare or random, effects. It is assumed that the majority of possible unexpected effects have been observed during the previous ESC-A flights.

At the beginning of the development process, flight data have been used to derive and fit the basic equations from in-flight measurements of pressure slopes. After that, the modification parameters have been identified and optimized using several optimization approaches. Hereafter, the results of this development process are presented.

During a depressurization phase $(n>0)$, the ullage pressure $P_{i}$ for timestep $i$ is depending on a number of different time- and event-correlating factors. The depressurization slope can be described by following equation:

$$
P_{i}=P_{0}\left(C_{\ddot{x}} M_{n, t} S_{d}\right)\left[\left(d_{1} e^{d_{2} \Delta t_{i}}+d_{3} e^{d_{4} \Delta t_{i}}\right)-d_{5}\right] .
$$

The ullage pressure at timestep $i$ is defined by the ullage pressure $P_{0}$ at $t=0$ (start of depressurization) and several statistical modifiers. $C_{\ddot{x}}$ is a factor describing the stage acceleration with the assumption that the acceleration leads to propellant settling and, thus, to a higher evaporation rate due to better coupling, less ullage cooling due to reduced liquid-gas interface and bubble transport during boiling. As a consequence for the model, the factor reduces the pressure loss during acceleration and varies between $C_{\ddot{x}}=1$ for the non- and $C_{\ddot{x}}>1$ for the accelerated states.

The nozzle mass flow factor $M_{n}$ represents the correlation between nozzle mass flow, flow speed, and number of activated nozzles $n$, driven by the system specific parameters (friction, pipe length, temperature, and diameters). The respective numbers for 1 to 12 parallel activated nozzles are defined on the system side and are taken from a look-up table.

Finally, the depressurization factor $S_{d}$ covers the reduction in liquid residuals due to evaporation and blowout. Since the total mass of the residual liquid is not constant for different missions, the factor is only assumed to be mission time dependent with $S_{d}=1$ for $t=0$ and $S_{d}<1$ for $t>0$. The factors $d_{1}$ to $d_{4}$ are derived from the initial setup of the depressurization equation and are optimized first before all other parameters. Factor $d_{5}$ biases a slight offset in the starting parameter since for small $t$, the equation returns $P_{i}>P_{0}$.

During a regeneration phase $(n=0)$, the ullage pressure $P_{i}$ for timestep $i$ can be described by the following equation:

$$
P_{i}=P_{0} S_{r}\left(r_{1} e^{r_{2} \Delta t}+r_{3} e^{r_{4} \Delta t}\right) .
$$


The regeneration factor $S_{r}$ covers again the reduction in liquid residuals due to evaporation and blowout. The factor is again only assumed to be mission time dependent with $S_{r}=1$ for $t=0$ and $S_{r}<1$ for $t>0$. The factors $r_{1}$ to $r_{4}$ are derived from the initial setup of the regeneration equation and are optimized first before all other parameters. During development, it was observed that the impact of acceleration on the regeneration rate can be neglected.

\subsection{Model Parameter Optimization}

With the setup of the simplified pressurization model, several unknown variables are introduced and have to be fitted to the flight data. Since the given 18 parameters are not well known, they will undergo several optimization loops. The basic aim of these loops is to generate a model that is able to reproduce the pressure slope for a given SCAR activation profile without taking into account the effects caused by potential sloshing effects.

The optimization is considered successful when the mean deviation between the slopes of the measured tank ullage pressure and the respective predicted pressure reaches a minimum. All deviations between model and physical measurement are then considered to be caused by modeling- or sloshing effects and will be treated later. To optimize the prediction model, a Monte-Carlo-based multiple stage simplex algorithm is used. The regular break criterion is set by a deviation corridor and has to be adapted iteratively.

\subsection{Model Performance}

After performing the optimization loop, the overall model precision for 34 ESC-A flights is $4.43 \% \pm 3.18 \%$. The overall calculation time for one simulation is between 0.7 and $0.95 \mathrm{~s}$ depending on the number of SCAR activations and ballistic phase durations.

Several observations have been made. The most important finding is that eventual sloshing of the liquid residuals in the tank takes place on a scale, which is well covered by the statistical analysis of the re- and depressurization slopes. Figure 7 illustrates exemplarily the $\mathrm{LH}_{2}$ tank ullage pressure calculated by the statistical approach in comparison to the flight measurements for a ballistic flight. Even if the initial sloshing event and the pressure slope during the ballistic phase are well covered by the simplified model, a single excessive pressure drop after $\sim 150 \mathrm{~s}$ leads to an offset. The maximum deviation for this flight is $\sim 200$ mbar.

Only on two instances, a macroscopic impact of liquid sloshing on the tank ullage pressure could be observed. For nonstandard missions, the performance of the algorithm is rather good due to the statistical character of the parameters. 

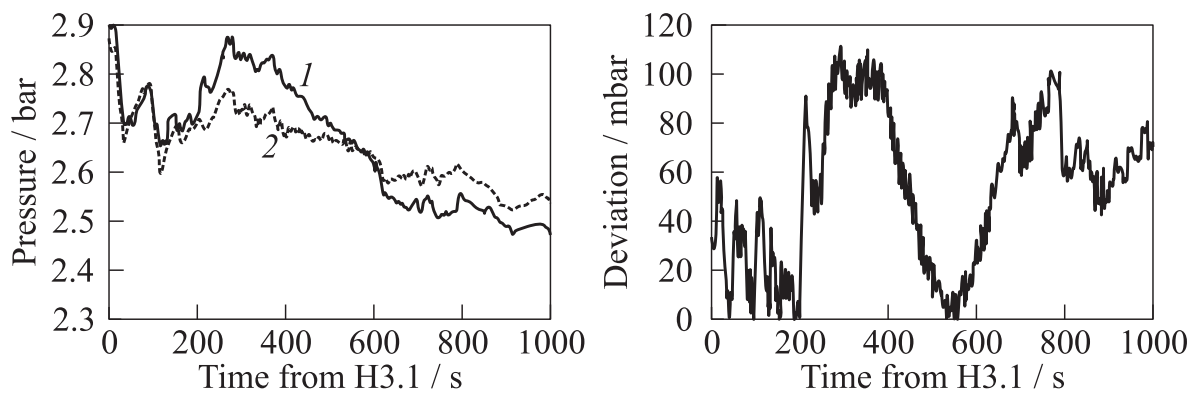

Figure 7 Deviations of the statistical MATLAB tool (1) to flight data L555 (2)

\section{EcosimPro PERFORMANCE MODEL}

EcosimPro is a simulation environment designed by Empresario Agrupados International for modeling physical processes based on differential-algebraic equations and discrete events. The modeling process is based on the EcosimPro modeling language EL which is used to design various types of single components that can be connected to a complex physical system. Due to the limitation to zeroand one-dimensional models, complex systems with long physical time durations, such as the ballistic flight phase of the upper stage, require a system analysis tool with limited dimensions and discretization.

\subsection{Model Setup}

The EcosimPro model of the ballistic phase aims to give a realistic view of the propellant behavior in the tanks during the ballistic flight. Therefore, the $\mathrm{LH}_{2}$ cold gas system has to be simulated with respect to variable heat loads and sloshing effects due to the upper stage maneuvers. The model is set up using the ESPSS library [4] at version 2.4 under EcosimPro 5.4 and consists of the tank model which takes account of the sloshing effects, the feed line system with its hydraulic boundary conditions, several time dependent valve control regulators, and the SCAR system thrusters. Tank walls and insulation layers define thermal capacities and conductivities of the tank and, thus, heat fluxes induced by a time variable thermal environment (Fig. 8).

Sensitivity analyses have identified a significant impact of the boiling model parameters on the depressurization and pressure regeneration behavior during the first sloshing event after engine flameout. Boiling as well as condensation effects are respected in the tank component. The time step size is adjusted by the transient solver, depending primarily on the thruster activation events. 


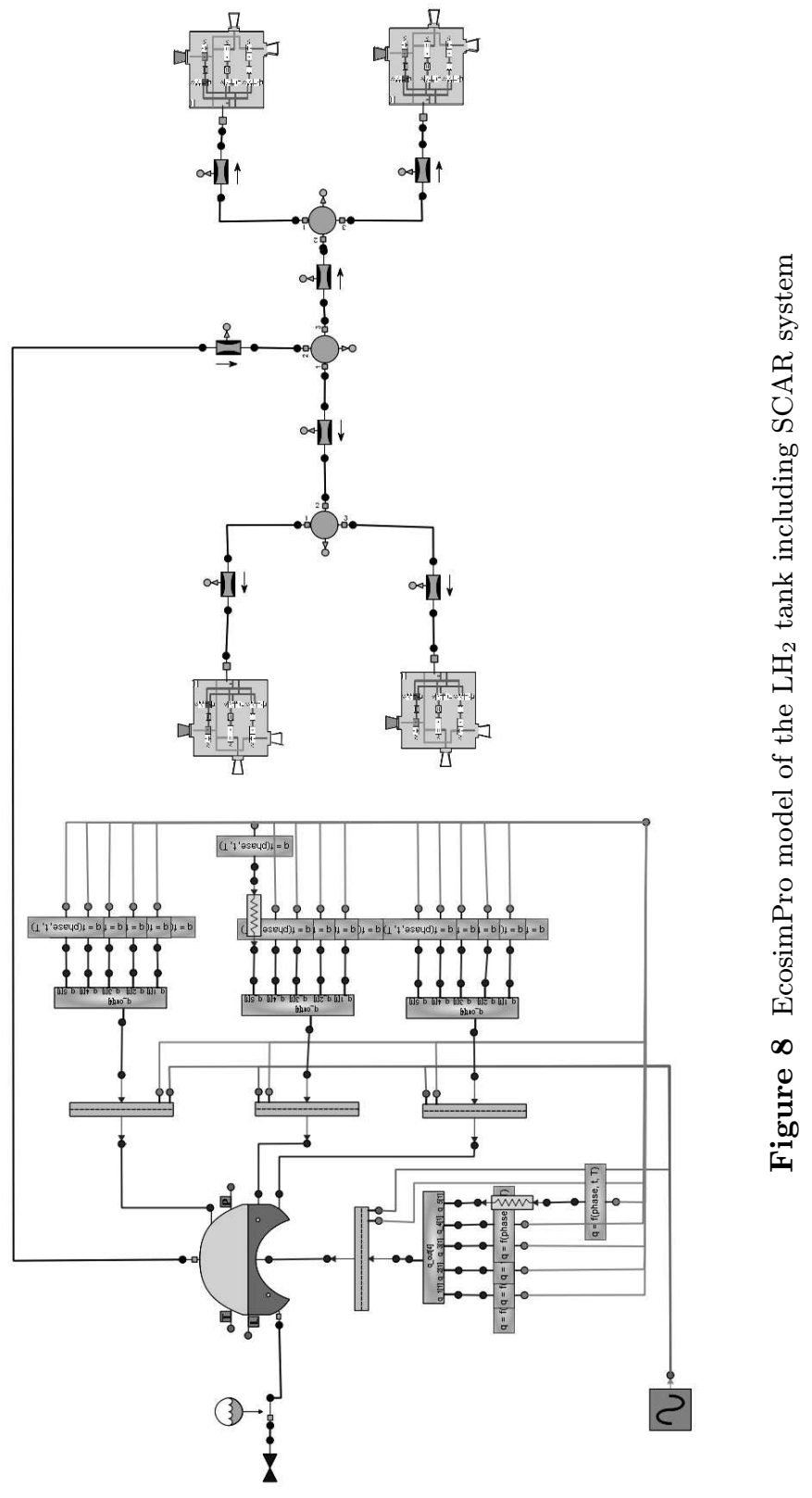




\subsection{Boundary Conditions}

The numerical model for ballistic phase analysis requires a set of boundary conditions. Heat fluxes are taken from the generic thermal environment, which includes sink temperatures, solar and albedo fluxes specific for each tank part. These are the upper dome (exposed to stage structure), cylindrical part and lower dome (deep space / Earth / Sun) and inner dome (LOx tank). They are depending on time and generic thermal scenario (hot, reference, and cold cases).

The simulation starts at engine shutdown. Thus, the residual mass with pressure and temperature of the liquid and gas phase are inputs. Thruster activations of the SCAR define the outgoing mass flow. In addition, the accelerations of the stage are used as input and are required to compute the heat transfer coefficients.

The wetted surfaces between liquid and gas (LG) and liquid and wall (LW) are computed by the use of the CFD tool Flow3D based on the SCAR activation profiles, respectively, torques, the stage mass, and inertia as well as the propellant residual mass [5]. According to Fig. 6, these 3D CFD results are inputs of the EcosimPro system model.

\subsection{Implementation of Three-Dimensional Data in the One-Dimensional System Model}

In the Flow3D CFD tool, the propellant tank is represented by a $3 \mathrm{D}$ geometry and a Cartesian numerical mesh. In consequence, the CFD analysis output, linked to the numerical discretization, has a 3D format as well. Each mesh cell contains the information of its position in the propellant tank and for each of the two interfaces (LG and LW) the wetted surface fraction for each cell face. This set of output values is provided for each numerical cell at each analysis time step.

In contrast to the 3D Flow3D format, the system model in EcosimPro is limited to a 1D discretization. The propellant tank is divided into slices along the tank symmetry axis, representing the numerical nodes. For the transformation from three dimensions to one dimension, a MATLAB script is used.

The LG interface areas of each CFD cell are summed up for each time step. For the LW interface, the CFD cells have to be assigned to the EcosimPro node according to their position on the tank symmetry axis. For each EcosimPro node and each time step, the sum of the corresponding CFD values is built. In consequence, the full set of $3 \mathrm{D}$ values is reduced to a single value for the $\mathrm{LW}$ wetted surface area for each EcosimPro node and one single value for the LG interface area for the complete fluid compartment. 

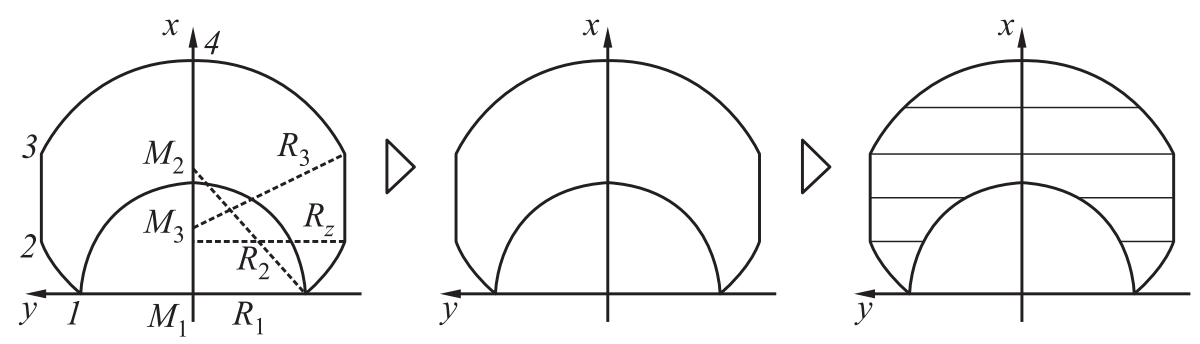

Figure 9 Transformation of 3D CFD results into 1D EcosimPro model inputs

Figure 9 presents a schematic illustration of the transformation of 3D CFD data into the 1D EcosimPro model format. The complex $\mathrm{LH}_{2}$ tank geometry, comprising tank internal equipment such as pressurization line and draining collector, is simplified to a numerical model (picture at the left-hand side). The 3D sloshing analysis is performed by an appropriate CFD model using a Cartesian numerical mesh (picture in the center). All 3D sloshing data are transformed to fit the sliced EcosimPro model (picture on the right-hand side).

\subsection{Model Parameter Correlation}

Even if the boundary conditions are well-defined, a large set of model inherent parameters affects the accuracy and robustness of the numerical solution. The EcosimPro ESPSS tank component applies an empirical boiling model covering the pool boiling regimes defined by the Nukiyama curve. Sensitivity analyses have highlighted the impact of these empirical boiling model parameters on the depressurization and regeneration rates.

Thus, correlation loops with four representative ESC-A flight data measurements are performed. Therefore, four Ariane 5 ESC-A launches were selected, representing a family of ballistic phase mission profile. Especially, the $\mathrm{LH}_{2}$ pressure evolution and cold gas system activations are similar. An additional mission with an extended ballistic phase (V173/L533) is also considered.

Parameters for the major sloshing phase (engine shutdown $+250 \mathrm{~s}$ ) were optimized regarding the hot, cold, and reference cases. Since the exact heat fluxes remain unknown, they can only be estimated by exposure time to sun radiation. Nevertheless, best correlations were reached for the hot case. These optimizations of the boiling parameters $C_{\mathrm{sf}}, f_{\mathrm{boi}}$, and $r$ from the Rhosenowcorrelation [6] were done manually:

$$
\dot{q}=\frac{\eta_{l} \Delta h_{v}}{L_{b}}\left(\frac{c_{\mathrm{pl}}\left(T_{w}-T_{s}\right)}{\Delta h_{v} \operatorname{Pr}_{l}^{f_{\mathrm{boi}}} C_{\mathrm{sf}}}\right)^{1 / r} .
$$


A reasonable implementation of fluid velocities calculated by CFD sloshing analyses did not show improvements. It seems that the implementation methodology affecting the heat transfer mechanisms do not match the real $\mathrm{LH}_{2}$ movements. Thus, a common set of boiling parameters was computed by the optimization approach without velocity implementation. Consequentially, further investigations could decrease deviations which are already below $5 \%$.

\subsection{Model Performance}

The time needed for the simulation of one flight (from engine shutdown until stage passivation) is between 3600 and $8000 \mathrm{~s}$ depending on the mission duration. Partly simulated scenarios (e.g., only sloshing phase) are possible for a known initial status. It has to be noted that the total analysis duration is driven by the complex CFD sloshing analysis providing the required EcosimPro inputs, not covered by the stated simulation duration.

For the complete ballistic phase, the deviations are below 5\%. In Fig. 10, the numerical analysis by EcosimPro and the flight measurement are compared for one exemplary flight. The first $200 \mathrm{~s}$, which are in the range of model parameter optimization, reflect the flight measurements very well. Even if the calculated pressure evolution has an excessive pressure regeneration after $200 \mathrm{~s}$, the maximum deviation is below 120 mbar for this flight.

The flight prediction accuracy by EcosimPro is affected by the quality of the required input data. Even if the CFD sloshing analysis, based on SCAR activation profiles, delivers flight specific data, other inputs are based on generic definitions (e.g., thermal environment). To further increase the accuracy and robustness of the EcosimPro flight prediction model, additional analysis and workarounds for nonstandard missions have to be performed. Additional flights with an extended ballistic phase will be a source of enhanced model correlations.
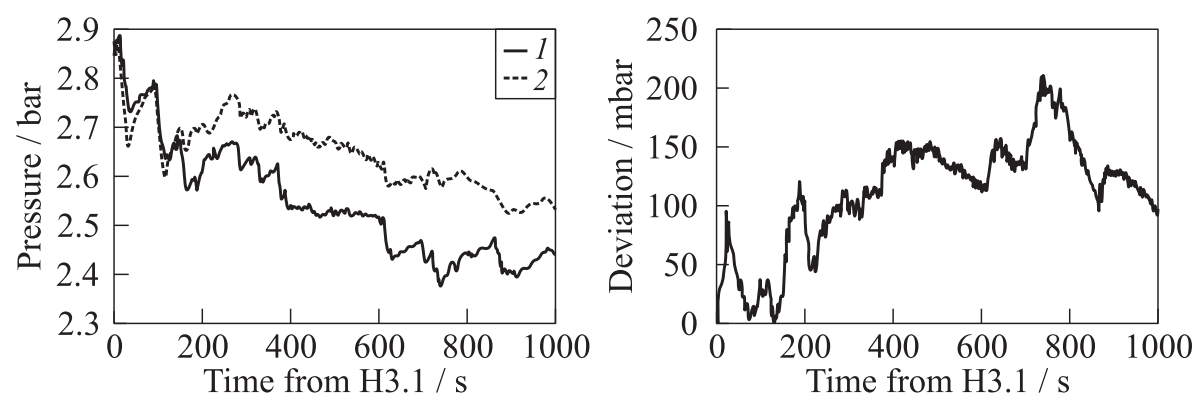

Figure 10 Deviations of the EcosimPro model (1) to flight data (2) 


\section{CONCLUDING REMARKS}

A performance model is required to ensure sufficient cold gas attitude control performance during long ballistic phase, e.g., for future launchers. Due to the coupling between thruster activation sequence, stage kinematics, and thermal environment, a dedicated work flow with iteration loops involving several tools and engineering disciplines was established.

To increase the performance of the initial coherence loop, a simplified model was implemented in MATLAB. The depressurization and regeneration due to sloshing was considered by a statistical approach limiting the number of required inputs. For standard mission phases, covered by flight experience, this model shows a sufficient accuracy by short calculation times.

For final and detailed analysis, an EcosimPro model comprising all relevant boundary conditions was set up. Especially, the implementation of 3D propellant sloshing effects (extracted from CFD calculations) into the 1D system model leads to a significant increase of accuracy and robustness also for nonstandard missions and future launcher design. For further improvements of analysis accuracy, additional correlations and sensitivity analyses with flight measurements will be performed in future.

\section{PROPOSAL OF FUTURE ACTIVITIES}

In addition to the already mentioned extension of underlying flight measurements, further areas of improvement are identified for ballistic phase performance prediction modeling.

For flight prediction, generic data, e.g., hot, reference, and cold cases for the thermal environment, are defined and applied. These generic definitions cover all probable mission scenarios and ensure a high level of confidence by a limited analysis effort. However, the application of these worst case conditions, usually, does not represent the real flight conditions. Here, simplified models for more detailed flight condition prediction and input generation are required.

As shown in Eq. (1), the heat transfer mechanisms have a significant impact on the $\mathrm{LH}_{2}$ evaporation and, in consequence, pressure regeneration. During the ballistic phase, the upper stage is under microgravity condition. In case of cold gas thruster activation (e.g., S34), acceleration occurs. Furthermore, the propellant movement due to sloshing overlays the empirically determined heat transfer coefficients. It might be reasonable that a more detailed consideration of heat transfer mechanisms of sloshing liquids under different acceleration conditions is required.

During the whole performance prediction process, interfaces between different engineering disciplines and tools exist. In the frame of the analysis work flow 
definition, the interfaces and data exchange formats are already defined (cf. Fig. 6). MATLAB scripts are used for data treatment reducing the data handling effort. An integrated system analysis approach, integrating all relevant tools and covering the data handling in-between, would decrease the analysis loop duration and would further increase the performance prediction accuracy and robustness.

\section{ACKNOWLEDGMENTS}

This work is funded by the German Federal Ministry of Economic Affairs and Energy (contract code 50 RL 1220) and administered by the German Space Agency (DLR). The presented activities are part of the Program to Enhance Upper Stage Performance and Reliability for Future Expendable Launchers (PREPARE). The authors would like to thank for the support fostering the competences of ballistic phase performance analysis as well as for supporting the development of numerical analysis tools.

Furthermore, thanks to Dr. Armin Isselhorst for his helpful consulting in the frame of EcosimPro modeling and to Patricia Netzlaf for performing the Flow3D analyses.

\section{REFERENCES}

1. Arndt, T. 2008. Stratification and destratification of liquid nitrogen $\mathrm{LN}_{2}$ in a cryogenic tank. 37th COSPAR Scientific Assembly. Montréal, Canada.

2. Van Foreest, A. 2014. Modeling of cryogenic sloshing including heat and mass transfer. Dissertation. Bremen.

3. Isselhorst, A., and K. Bergemann. 2009. Ballistic flight phase simulation with euces Tool for ESC-A upper stage. AIAA Paper No. 2009-5810.

4. Moral, J., R. Pérez, J. Steelant, and M. de Rosa. 2010. ESPSS Simulation Platform.

5. Fries, N., P. Behruzi, T. Arndt, M. Winter, G. Netter, and U. Renner. 2012. Modelling of fluid motion in spacecraft propellant tanks - sloshing. Space Propulsion Conference. Bordeaux.

6. Emprasarios Agrupados. 2012. User Manual of the ESPSS EcosimPro Libraries. Doc. $12205 / 07 / \mathrm{NL} / \mathrm{CP}$. TN-4140. 